\title{
Linear parametric hydrodynamic models for ocean wave energy converters identified from numerical wave tank experiments
}

\author{
Josh Davidson*, Simone Giorgi, John V. Ringwood \\ Centre for Ocean Energy Research, National University of Ireland Maynooth, Co. Kildare, Ireland
}

\section{A R T I C L E I N F O}

Article history:

Received 8 January 2014

Accepted 27 April 2015

Keywords:

Hydrodynamic modelling

Wave energy converters

Computational fluid dynamics

System identification

Optimisation

Linearisation

\begin{abstract}
A B S T R A C T
Mathematical modelling of wave energy devices has many uses, including power production assessment, simulation of device motion and as a basis for model-based control design. Apart from computationally heavy approaches, such as those based on computational fluid dynamics (CFD) and smooth particle hydrodynamics (SPH), the vast majority of models employed in the simulation and analysis of wave energy converters (WECs) are based on boundary-element methods (BEMs). While BEM models have been shown to be useful, they have the inherent limitation that they are linearised around the still water level, with validity only on the immediate vicinity of this equilibrium point. In this paper, we develop a new modelling methodology, which combines the fidelity of CFD models with the computational attractiveness of BEM-type models. This flexible methodology can give representative linear models, or be extended into the nonlinear domain, as desired.
\end{abstract}

(c) 2015 Elsevier Ltd. All rights reserved.

\section{Introduction}

Mathematical system modelling provides a possibility to determine an abstract description of a physical system, which can be easily subsequently manipulated, analysed and simulated on a computer. This can help to expedite the process of system design, while significantly reducing the cost of building physical prototypes at various scales. In addition, mathematical models are required as the fundamental building block upon which model-based control design is performed. However, if the analysis and results emanating from these models are to be meaningful, the models themselves must be a reasonably faithful representation of the original physical system.

There is a significant motivation to work with linear models. They are computationally simpler, obey superposition (divide and conquer) and lend themselves to a vast array of mathematical tools which can be used for their analysis and simulation. It is accepted practice in many disciplines, such as control engineering, that many systems are linearised around an operating point. In control systems, this is normally a reasonable assumption, since the usual control objective is to drive a system to a specific setpoint. However, in the contrasting case of wave energy, the objective is to drive the system as far away from equilibrium as possible. This is likely to result in the excitation of nonlinear dynamics, resulting in non-representative linear models.

Typically, linear hydrodynamic models based on boundary element methods are employed (Li and Yu, 2012; Maguire, 2011)

\footnotetext{
* Corresponding author.

E-mail address: jdavidson@eeng.nuim.ie (J. Davidson).
}

for WECs, with hydrodynamic parameters determined from frequency-domain codes such as WAMIT or AQUAPLUS or, in the time domain using ACHIL-3D. These essentially follow a physical (firstprinciples) approach in parameterising Cummins equation, using finite element methods, where model-order reduction techniques (Taghipour et al., 2008) can be used to deliver a finite-order linear model. In some cases, such models can be extended to include some nonlinear effects such as viscous damping, for example using the Morison equation (Morison et al., 1950), where the viscous damping coefficient is determined based on historical experience. An alternative determination of the viscous damping coefficient is that by Bhinder et al. (2012), where CFD is used to evaluate the viscous force, to which a viscous damping constant is fitted. BEMs have also been used to parameterise nonlinear models (Gilloteaux, 2007; Gilloteaux et al., 2008), but require the recalculation of hydrodynamic parameters (on the instantaneous wetted surface) at each sampling instant, with a resulting high computational overhead.

An alternative modelling approach, popular in the systems and control community, is that of system identification, where models are determined from input/output data measured from the system under study (Ljung, 1999). Such methods are particularly useful where the system to be modelled is very complex and/or does not easily lend itself to first principles modelling. However, one major difficulty in system identification is ensuring that the input/output data used to determine the model is sufficiently representative of the system dynamics and, in particular, must cover the range of frequencies and amplitudes likely to be encountered during system operation. In the WEC case, such a range of excitation signals are not likely to be 
available in the open ocean (at least not in a reasonably short time frame) and there are difficulties in exactly enumerating the excitation experienced by the device, particularly for a directional device. In short, there is no external control of the excitation. One other possibility is to employ tank tests. However, in addition to the significant cost and the need for a physical prototype, there may be limitations on the range of excitation signals available and tank wall reflections may further limit the range and duration of viable tests.

One possibility for generating suitable input/output data is to use a numerical wave tank (NWT), implemented in CFD, which has the following advantages:

- Reflections from 'tank' walls can be effectively controlled.

- Can test the device at full scale, eliminating scaling effects.

- A wide variety of excitation signals, including incident waves and forces directly applied to the device, as well as free response tests, can be implemented.

- The device can be constrained to different modes of motion without requiring mechanical restraints which can add friction and alter the device dynamics.

- Signals can be passively measured without requiring physical sensor devices which can alter the device or fluid dynamics and are subject to measurement error, and most importantly.

- Specialist equipment, including a prototype WEC device, is not required.

Though CFD codes are relatively inexpensive (for example the opensource OpenFOAM code), they are computationally heavy and are best run on high-performance computers (HPCs). However, HPCs are now becoming quite cost effective.

Adopting a system identification approach also offers considerable flexibility in model parameterisation and the relationship to physical quantities and the desired complexity/fidelity trade-off. Regarding the connection with physical quantities, the following general classes are recognised:

- White-box, where each parameter represents a physical quantity.

- Grey-box (and the sub-classes of off-white, smoke-grey, steelgrey and slate-grey Ljung, 2008), with various levels of connection to the underlying hydrodynamical structure.

- Black-box, where the model simply reproduces the experimental output data, given the same stimulus, but the internal model structure bears no resemblance to the physical world.

White and grey box models present the significant benefit of a structure which is well related to physical aspects of the system and the model variables usually represent physical quantities. As the shade of grey gets darker, the connection with the physical world diminishes, until the only connection of black-box models with the physical world is the representation of the overall model input and output. For the current study, focus will be on grey-box modelling, as we try to retain the (physical) structure of a Cummins-type equation, while employing system identification techniques to get a good fit of the model to the NWT response data.

In this paper, we present a new methodology for the development of hydrodynamic models for WECs, outlined in Fig. 1. For this particular study, we will focus on the development of linear hydrodynamic models, in order to allow a comparison with linear models parameterised using BEM methods, and show the possibility to determine representative linear models valid for different wave heights and their interrelationship.

The remainder of the paper is laid out as follows: Section 2 describes the salient points of the numerical wave tank implementation, while Section 3 provides the linear WEC modelling background needed. Section 4 details the means by which the parameters of the

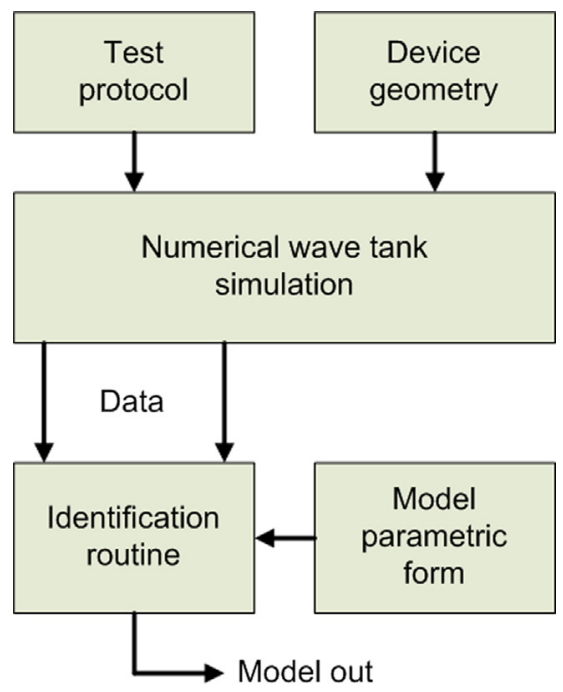

Fig. 1. Overview of modelling methodology.

linear hydrodynamic models are determined and Section 5 documents a case study showing the results of such a procedure for the case of a heaving buoy WEC. Finally, conclusions are drawn in Section 6.

\section{Numerical wave tank}

Numerical wave tanks (NWTs) have been used for many decades in ocean engineering to analyse fluid-structure interaction (Tanizawa, 2000). The fluid dynamics are governed by the transfer of mass, momentum and energy. These three processes are described by a set of nonlinear partial differential equations, known as the Navier-Stokes equations, detailed as follows:

1. Continuity equation:

$$
\frac{\partial \rho}{\partial t}+\nabla \cdot(\rho \mathbf{u})=0
$$

2. Momentum equation:

$\rho \frac{\partial \mathbf{u}}{\partial t}=\nabla \cdot \tau_{i j}$

3. Energy equation:

$$
\rho \frac{\partial e}{\partial t}=\nabla \cdot(k \nabla T)-p \nabla \cdot \mathbf{u}+\tau_{i j}^{\nu} \frac{\delta u_{i}}{\delta x_{j}} .
$$

where $\rho$ is the fluid density, $\mathbf{u}$ is the velocity, $e$ is the internal energy, $T$ is the temperature, $k$ is the thermal conductivity and $\tau_{i j}$ is the stress tensor comprising the pressure, $-p \delta_{i j}$, and viscous terms, $\tau_{i j}^{v}$

$\tau_{i j}^{v}=\mu\left\{\frac{\delta u_{i}}{\delta x_{j}}+\frac{\delta u_{j}}{\delta x_{i}}\right\}+\delta_{i j} \lambda \nabla \cdot \mathbf{u}$.

where $\mu$ is the coefficient of viscosity, $\delta_{i j}$ is the Kronecker delta function and $\lambda$ is the bulk viscosity.

The coupled continuity, momentum and energy equations, Eqs. (1)-(3), are indeterminate and require two more equations to obtain closure which are provided by the ideal gas laws

$p=\rho R T$, 
and

$e=c_{v} T$.

where $c_{v}$ is the specific heat at constant volume.

In general, these equations have no known analytical solution; however they may be numerically discretized to obtain a solution requiring very high levels of computation. Traditionally, such levels of computation were infeasible, necessitating many linearising assumptions such as inviscid, irrotational and incompressible fluids to allow a computationally tractable solution, whereby NWTs were implemented based on the linear theory of the velocity potential and boundary integral equations. However, with the continuing increase in computer power, the full Navier-Stokes equations can be implemented using computational fluid dynamics (CFD).

To solve the Navier-Stokes equations, CFD discretises the continuous partial differential equations into a system of linear algebraic equations that can be solved on a computer. That is, the continuum is broken up into finite temporal and spatial portions to transform a calculus problem into an algebraic problem. This is implemented spatially on a mesh with different methods used to discretise the spatial volumes such as finite volume, finite difference and finite element approaches. The problem is then also discretised temporally using individual time steps.

CFD can be used to simulate fluid-structure interactions following the process outlined in Fig. 2. In the first step, the values for the pressure and velocity field are solved everywhere in the fluid domain, where the presence of the body introduces a boundary condition in the solution. The hydrodynamic pressure can then be integrated over the surface of the body to yield the hydrodynamic force. The resulting body motion is calculated and then the simulation iterates forward in time and returns to the first step. In this paper, we seek to extract all the information from this process and condense it into a representative linear model.

The modelling methodology presented in this paper will utilise NWT free decay experiments, simulated in CFD, to generate the data for the system identification process. It is important to note that a CFD simulation requires knowledge of a body's mass in order to calculate its motion, so we assume it as a known property of the WEC being investigated.

\subsection{NWT free decay experiment}

In a free decay experiment, a body is given an initial amount of potential energy by displacing it against a restoring force away from its equilibrium position. The body is then released from this displaced position and will begin to move back towards its equilibrium position converting the potential energy to kinetic energy whilst also dissipating energy due to damping effects from the surrounding fluid. Depending on the level of damping, the body will typically oscillate around its equilibrium position until all the energy has been dissipated and it comes rest at equilibrium. For the heave, pitch and roll

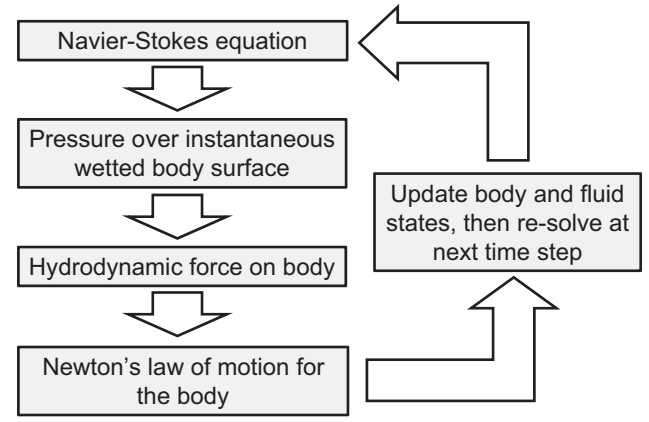

Fig. 2. Schematic of CFD process for fluid-structure interaction. modes of motion, the mismatch between the gravitational and buoyancy forces acts as the restoring force. The surge, sway and yaw modes have no natural restoring forces; however free decay experiments can still be performed in NWTs for these modes by defining a linear spring force to act on the body in surge, sway and yaw.

The free decay test, which requires no exogenous input, allows the identification process to focus only on the parameters connected with the inertia, restoring and damping forces. The output signals from this experiment, which feed into the parameter identification, are the body's position and the hydrostatic force acting on the body.

\section{Linear models for wave energy converters}

The fluid-structure interaction between WECs and the ocean is described by a hydrodynamic model. In their systematic review of hydrodynamic modelling methods for point absorber WECs, Li and Yu (2012) show that these methods evolved from the hydrodynamic modelling of ships and offshore floating structures. An excellent description and comparison of the different hydrodynamic modelling methods for the dynamic response of marine structures is given by Taghipour et al. (2008). At the heart of these modelling methods is the Cummins equation derived in 1962 (Cummins, 1962).

\subsection{Cummins equation}

By considering the hydrodynamic radiation of a body, with zero forward speed, in an ideal fluid, Cummins (1962) showed that the radiation forces can be expressed as

$f_{R}(t)=m_{\infty} \ddot{y}(t)+\int_{-\infty}^{t} h_{R}(t-\tau) \dot{y}(\tau) d \tau$

where $y(t)$ is the position of the body, $m_{\infty}$ is the high-frequency limit of the added-mass and $h_{R}(t)$ is the impulse response function. The shape of the body's wetted surface determines the hydrodynamic radiation force felt by the body when it moves in the fluid. The transfer function between the body's velocity and the radiation force is the radiation impedance

$Z(i \omega)=\frac{F_{R}(i \omega)}{\dot{Y}(i \omega)}=N(\omega)+i \omega m_{a}(\omega)$.

The real part of the radiation impedance is the radiation resistance, $N(\omega)$, and the imaginary part the product of the frequency and the added mass, $m_{a}(\omega)$. At infinite frequency, the added mass tends to the finite constant, $m_{\infty}$, which is subtracted from the radiation impedance to form the reduced radiation impedance (to avoid divergence issues in the convolution integral of Eq. (7))

$H_{R}(i \omega)=N(\omega)+i \omega\left[m_{a}(\omega)-m_{\infty}\right]$.

The inverse Fourier transform of $H_{R}(i \omega)$ is the impulse response function, $h_{R}(t) . H_{R}(i \omega)$ and $h_{R}(t)$ satisfy the following properties (Pérez and Fossen, 2008):

$\lim _{\omega \rightarrow 0} H_{R}(i \omega)=0$

$\lim _{\omega \rightarrow \infty} H_{R}(i \omega)=0$

$\lim _{t \rightarrow 0^{+}} h_{R}(t) \neq 0$

$\lim _{t \rightarrow \infty} h_{R}(t)=0$

$N(\omega) \geq 0 \quad \forall \omega \quad$ (from passivity) 
In addition to the radiation force, the body experiences a (linearised) hydrodynamic restoring force:

$f_{S}(t)=-K y(t)$

where the restoring coefficient $K \geq 0$. Considering an external excitation force $f_{e}(t)$, the complete Cummins equation for a body with mass $M$ is specified as

$\left(M+m_{\infty}\right) \ddot{y}(t)+\int_{-\infty}^{t} h_{R}(t-\tau) \dot{y}(\tau) d \tau+K y(t)=f_{e}(t)$.

\subsubsection{Possible extensions}

The Cummins equation in Eq. (16) can be easily extended to matrix form for bodies with multiple degrees of freedom, multiple components and/or multiple bodies in a WEC array. When modelling WECs, it is possible to extend the Cummins equation to include linear/ nonlinear mooring forces and a linear/nonlinear power take-off (PTO) term as part of the external force on the right hand side of Eq. (16). A common approach to also include the viscous drag effect to the nonviscous hydrodynamic forces is to add a quadratic damping term, via the Morison equation (Li and Yu, 2012). Additional possible extensions of the model structure include the introduction of a nonlinear restoring force and/or a nonlinear Froude Krylov force, though the latter results in a significant increase in complexity and computational requirements (Merigaud et al., 2012).

\subsubsection{State space representation}

There are a number of ways to implement the Cummins equation numerically; in the present work the state-space method is used. The convolution integral in the Cummins equation typically makes it difficult to use; however the state space method easily handles it through approximating the convolution integral by a finite-order system of differential equations with constant coefficients. Specifically,

$\int_{-\infty}^{t} h_{R}(t-\tau) \dot{y}(\tau) d \tau$

can be represented by the following state space sub-system

$\dot{\mathbf{x}}_{\mathbf{s}}(t)=\mathbf{A}_{\mathbf{s}} \mathbf{x}_{\mathbf{s}}(t)+\mathbf{B}_{\mathbf{s}} \dot{y}(t)$

$v_{s}(t)=\mathbf{C}_{s} \mathbf{x}_{\mathbf{s}}(t)$

where $\mathbf{x}_{\mathbf{s}}(t)=\left[x_{s 1}(t) x_{s 2}(t) \cdots x_{s n}(t)\right]^{T}$ is the state vector. The impulse response function of this state space is (Furuta et al., 1988)

$g_{R}(t)=\mathbf{C}_{\mathbf{s}} e^{\mathbf{A}_{\mathbf{s}} t} \mathbf{B}_{\mathbf{s}}$

and the Laplace transform of $g_{R}(t)$ can be written as

$G_{R}(s)=\mathbf{C}_{\mathbf{s}}\left(s \mathbf{I}-\mathbf{A}_{\mathbf{s}}\right)^{-1} \mathbf{B}_{\mathbf{s}}$

$G_{R}(s)=\frac{b_{m} s^{m}+\cdots+b_{1} s+b_{0}}{s^{n}+a_{n-1} s^{n-1}+\cdots+a_{1} s+a_{0}}$

The convolution integral, Eq. (17), and the state space system of Eq. (18), are equivalent if $h_{R}(t)=g_{R}(t)$ (and consequently $H_{R}(s)=G_{R}(s)$ ). This exact equivalence holds only for a state-space system of infinite order (i.e. $n=\infty$ ); however, an approximation is generally made to represent the convolution integral with a state space system of finite order $n$, as

$\operatorname{Re}\left\{G_{R}(j \omega)\right\} \simeq N(\omega)$

and

$\operatorname{Im}\left\{G_{R}(j \omega)\right\} \simeq \omega\left[m_{a}(\omega)-m_{\infty}\right]$

$G_{R}(j \omega)$ must also satisfy the properties of $H_{R}(j \omega)$, Eqs. (10)-(14). A consequence of Eq. (10) is that $G_{R}(0)=0$; therefore $b_{0}=0$. From Eq. (11), it follows that $G_{R}(s)$ is strictly proper $(n>m)$ and, as a consequence of Eq. (12), $m=n-1$ (Perez and Fossen, 2011). From these constraints it follows that

$G_{R}(s)=\frac{b_{n-1} s^{n-1}+\cdots+b_{1} s}{s^{n}+a_{n-1} s^{n-1}+\cdots+a_{1} s+a_{0}}$

There are many possible realisations of a state space model; here the observable companion-form realisation is used, since it has the advantage of only requiring a small number of parameters (Kailath, 1980; Levine, 2011), with $\mathbf{A}_{\mathbf{s}}, \mathbf{B}_{\mathbf{s}}$ and $\mathbf{C}_{\mathbf{s}}$ of the following form:

$\mathbf{A}_{\mathbf{s}}=\left[\begin{array}{cccccc}0 & 0 & 0 & \ldots & 0 & -a_{0} \\ 1 & 0 & 0 & \ldots & 0 & -a_{1} \\ 0 & 1 & 0 & \ldots & 0 & -a_{2} \\ \vdots & \vdots & \vdots & \ddots & \vdots & \vdots \\ 0 & 0 & 0 & \ldots & 0 & -a_{n-2} \\ 0 & 0 & 0 & \ldots & 1 & -a_{n-1}\end{array}\right]$,

$\mathbf{B}_{\mathbf{s}}=\left[\begin{array}{llllll}0 & b_{1} & b_{2} & \ldots & b_{n-2} & b_{n-1}\end{array}\right]^{T}$,

$\mathbf{C}_{\mathbf{s}}=\left[\begin{array}{llllll}0 & 0 & 0 & \ldots & 0 & 1\end{array}\right]$

The Cummins equation, Eq. (16), can now be represented by the following state equation:

$\dot{\mathbf{x}}(t)=\mathbf{A x}(t)+\mathbf{B} f_{e}(t)$,

$y(t)=\mathbf{C x}(t)$,

where

$\mathbf{A}=\left[\begin{array}{cccccccc}0 & 0 & 0 & \ldots & 0 & -a_{0} & 0 & 0 \\ 1 & 0 & 0 & \ldots & 0 & -a_{1} & 0 & b_{1} \\ 0 & 1 & 0 & \ldots & 0 & -a_{2} & 0 & b_{2} \\ \vdots & \vdots & \vdots & \ddots & \vdots & \vdots & \vdots & \vdots \\ 0 & 0 & 0 & \ldots & 0 & -a_{n-2} & 0 & b_{n-2} \\ 0 & 0 & 0 & \ldots & 1 & -a_{n-1} & 0 & b_{n-1} \\ 0 & 0 & 0 & \ldots & 0 & 0 & 0 & 1 \\ 0 & 0 & 0 & \ldots & 0 & -\frac{1}{\mu} & -\frac{K}{\mu} & 0\end{array}\right]$,

$\mathbf{B}=\left[\begin{array}{llllll}0 & 0 & \ldots & 0 & 0 & \frac{1}{\mu}\end{array}\right]^{T}$,

$\mathbf{C}=\left[\begin{array}{llllll}0 & 0 & \ldots & 0 & 1 & 0\end{array}\right]$,

$\mathbf{x}(t)=\left[\begin{array}{lll}\mathbf{x}_{\mathbf{s}}(t) & y(t) \dot{y}(t)\end{array}\right]^{T}$,

where $\mu=\left(M+m_{\infty}\right)$. This linear hydrodynamic model is parameterised by $2 n+2$ parameters $\left(M, m_{\infty}, K, a_{0}, \ldots, a_{n-1}, b_{1}, \ldots, b_{n-1}\right)$.

The input to the model of Eqs. (29) and (30) is the wave excitation force, $f_{e}(t)$. It is often desirable to use the free surface elevation as an input, which can be achieved via an additional subsystem which takes as an input the wave elevation and outputs the excitation force (Yu and Falnes, 1995). This wave excitation force subsystem will have its own specific structure and parameters which can be identified using NWT experiments. However, since the current paper looks utilises only free response tests, the excitation force calculation is not included.

\subsection{Stability and passivity properties}

Now that the parametric structure is defined, it is necessary to find the constraints on the parameters to guarantee the properties of stability and passivity for both the sub and total systems. Indeed, the original physical system (a body floating on water) is characterised by the properties of stability and passivity (Pérez and Fossen, 2008) and, 
consequently, the utilised model has to inherit these properties to be able to produce accurate predictions.

In Perez and Fossen (2011), stability is not enforced as a constraint and consequently the resulting model from the least squares minimisation may not necessarily be stable. Their proposed solution after identification is the reflection of the unstable poles from the right-hand side to the left-hand side of the complex plane, thus obtaining a suboptimal solution. In the current work, new a priori constraints on the parameters are introduced in the optimisation to guarantee the stability of both sub-system and total-system for any order.

Applying the Routh Criterion (Power and Simpson, 1978), to the polynomial in the denominator of Eq. (25), it is possible to find analytical constraints on the parameters $a_{i}$ for $i=0, \ldots,(n-1)$. The stability of the total system $(\forall n)$ is guaranteed by introducing into the optimisation the nonlinear constraints $\operatorname{Re}\left\{P_{i}\right\}<0$, where $P_{i}$ for $i=1, \ldots,(n+2)$ are the poles of the total system.

Passivity describes an intrinsic characteristic of systems that can store and dissipate energy, but not create it. For linear-time-invariant systems, a necessary and sufficient condition for passivity is that the real part of the transfer function is positive for all frequencies (positive realness) (Pérez and Fossen, 2008). Since the Cummins equation, Eq. (16), has the structure of a negative feedback interconnection, and because a negative feedback interconnection of passive systems is passive (Perez and Fossen, 2011), the total state space equations (29) and (30) are passive iff the sub-state space equation (18) is passive (i.e. $\left.\operatorname{Re}\left\{G_{R}(i \omega)\right\} \geq 0 \forall \omega\right)$. In Pérez and Fossen (2008) and Perez and Fossen (2011), passivity of the sub-state space is tested by trying different order approximations and choosing a passive candidate. Here, we introduce new analytical constraints on the parameters to guarantee that $\operatorname{Re}\left\{G_{R}(i \omega)\right\} \geq 0 \forall \omega$.

\section{Model parameter identification}

In this section a methodology for identifying the parameters for the linear hydrodynamic model is outlined. The method utilises NWT free decay experiments, simulated in CFD, to generate the data for the system identification process. Again, we note that the present methodology does not identify the parameter $M$, rather assumes it as a known property of the WEC being investigated and can be determined from a variety of numerical modelling tools, such as AutoCAD. The free decay test focusses only the parameters relating to the inertia, restoring and radiation forces i.e. $\left(m_{\infty}, K, a_{0}, \ldots\right.$, $\left.a_{n-1}, b_{1}, \ldots, b_{n-1}\right)$.

\subsection{Identification procedure}

The procedure to utilise NWT generated data to identify a linear parametric model is based on two main steps:

- Selection of a model structure that provides a set of possible models dependent on the parameter values. In this case, the model structure is represented by Eqs. (29)-(34), and a key choice is the dynamical order of the radiation damping subsystem i.e. $n$.

- Selecting the specific model in the set which is most representative of the system dynamics by determining the values of the parameters from the information in the NWT generated data.

These two step are typical in system identification (Ljung, 1999). However, in contrast to traditional system identification, where forcing input signals are used to excite the system dynamics, we utilise only free decay experiments. We then employ a general optimisation routine to determine the parameters of the original continuous-time model, which is in contrast to most popular system identification methods, which use discrete-time models and accompanying identification methods specific to these model structures. While such discrete-time methods are computationally efficient, the retention of the continuous-time model format preserves the strong connection of the model parameters to physical quantities, while the general optimisation method provides a framework which is agnostic to the model form, allowing a very wide variety of both linear and nonlinear models to be employed. Fig. 3 shows the block diagram of the sequence of steps to estimate the linear model's parameters from NWT data.

\subsection{Restoring term estimate}

The restoring force, $f_{s}(t)=-K y(t)$ with $K>0$, arises from the mismatch between the gravitational force, $M g$, and the hydrostatic (buoyancy) force, $F_{H}(t)$,

$f_{S}(t)=F_{H}(t)-M g$.

The restoring force coefficient, $K$, can therefore be identified from knowledge of the hydrostatic force $F_{H}(t)$ and the body position $y(t)$. Fig. 4 shows an example of this where a restoring force obtained from NWT experiment is plotted as a function of displacement. The relationship between the restoring force and the body displacement is then approximated by a straight line passing through the origin with slope $-K$, using least squares on the NWT generated data

\subsubsection{Estimation of radiation and added mass terms}

The output of the system described by Eqs. (29) and (30) is the superposition of the zero-input component and the zero-state component, evaluated as

$y(t)=\mathbf{C} e^{\mathbf{A}\left(t-t_{0}\right)} \mathbf{x}\left(t_{0}\right)+\int_{t_{0}}^{t} \mathbf{C} e^{\mathbf{A}(t-\tau)} \mathbf{B} f_{e}(\tau) d \tau$

If $t_{0}=0$ and $f_{e}(t)=0 \forall t$, then

$y(t)=\mathbf{C} e^{\mathbf{A} t} \mathbf{x}(0)$

Eq. (37) describes the free decay oscillation of the floating body, where $x_{n+1}(0)$ represents the initial displacement of the body from its equilibrium position. Fig. 5 shows the methodology used to determine the values of the $2 n$ parameters, $\boldsymbol{\theta}=\left[a_{0}, \ldots, a_{n-1}, b_{1}, \ldots, b_{n-1}, m_{\infty}\right]$,

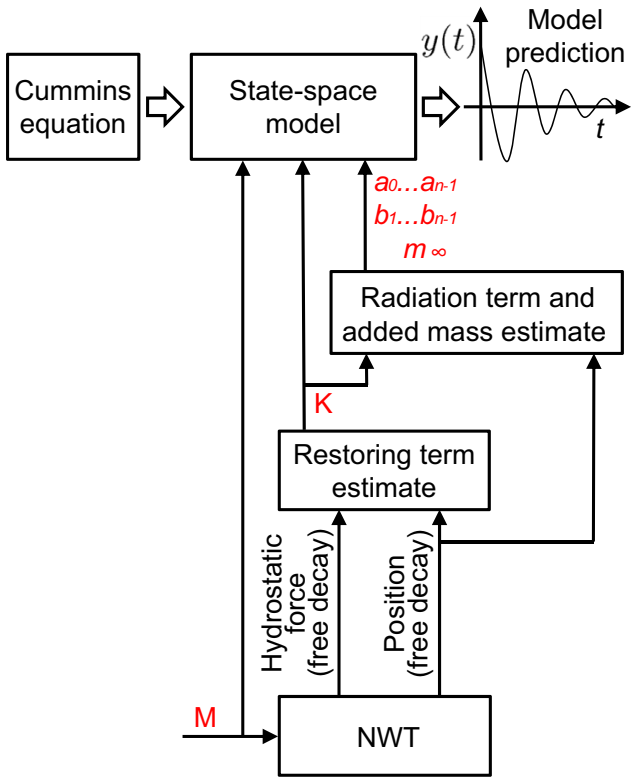

Fig. 3. Block diagram of the sequence of steps to estimate the linear system parameters from NWT data. 


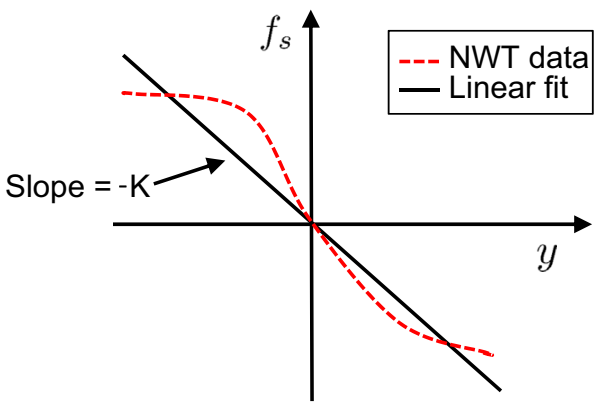

Fig. 4. Graphical representation of estimating the linear restoring force coefficient, $K$, from the restoring force versus position data obtained from a NWT experiment.

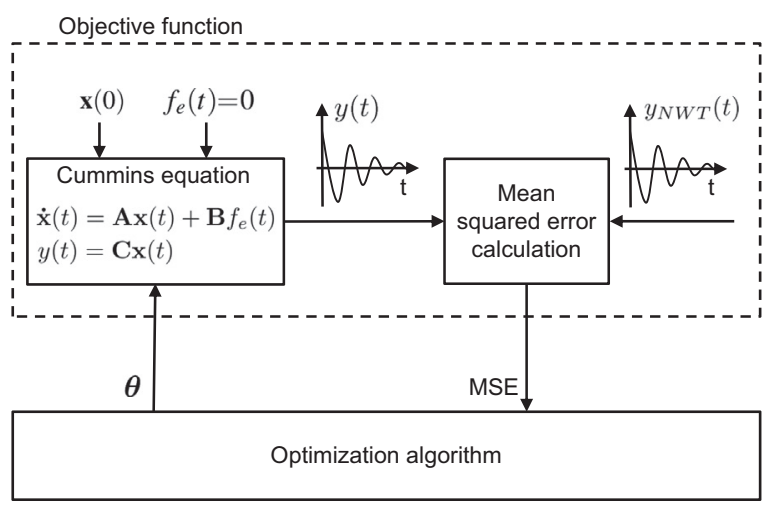

Fig. 5. Block diagram of the optimisation methodology to estimate the $2 n$ parameters $\boldsymbol{\theta}=\left[a_{0}, \ldots, a_{n-1}, b_{1}, \ldots, b_{n-1}, m_{\infty}\right]$. Because only free decay experiments are considered, the external force $f_{e}$ is set to zero.

where the zero-input response, Eq. (37), approximates the free decay NWT data via the criterion

$\hat{\boldsymbol{\theta}}=\arg \min \sum_{i} w_{i}\left|y_{N W T}\left(t_{i}\right)-y\left(t_{i}, \boldsymbol{\theta}\right)\right|^{2}$

where $y_{N W T}$ are the data generated from the NWT experiments, $w_{i}$ are the weighting coefficients (for simplicity, we use $w_{i}=1 \forall \mathrm{i}$ ), and $\hat{\boldsymbol{\theta}}$ are the parameters giving the best least squares fit, according to Eq. (38). The optimisation problem in Eq. (38) is nonlinear in the parameters, with a strong sensitivity to the initial seed $\boldsymbol{\theta}_{0}$, caused by an objective function with multiple local minima. To ensure that a good global solution to Eq. (38) is achieved, a concurrent search method is employed, which maintains a number of candidate solutions spread across the search space. In particular, we utilise the Matlab implementation of a genetic algorithm as a solver.

\section{Case study}

In this section, the methodology outlined in Section 4 is applied for the case of the heave motion of a floating cylindrical body with radius $0.5 \mathrm{~m}$ and draught $0.5 \mathrm{~m}$ (Fig. 6).

\subsection{Numerical wave tank experiments}

The NWT used in these experiments is simulated using the open source CFD software OpenFOAM. For finer detail regarding the development of a NWT in OpenFOAM, see Afshar (2010), Lambert (2012) and Cathelain (2013). The particular NWT used in these experiments is a modification of the NWT developed by Cathelain.

The geometry of the tank is a cylinder $5 \mathrm{~m}$ in height and $50 \mathrm{~m}$ in diameter, where the large diameter was chosen to eliminate the effect of wave reflection from the side walls of the tank. The tank is filled with $3 \mathrm{~m}$ of water with a density of $997 \mathrm{~kg} \mathrm{~m}^{-3}$ and the remaining

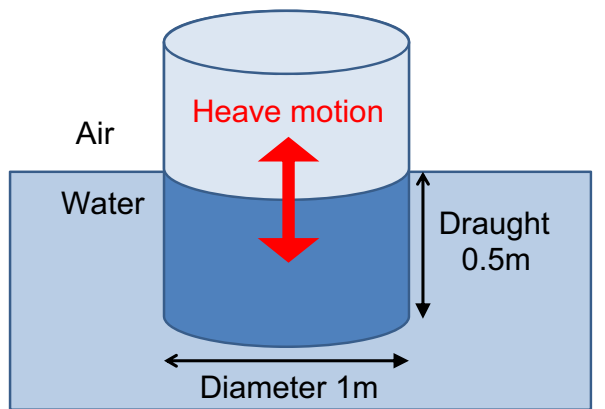

Fig. 6. The floating cylinder considered in the present case study.

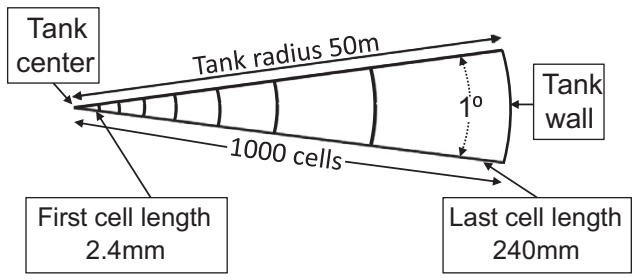

Fig. 7. Schematic top view of the NWT where axisymmetry has enabled the tank's circular cross-section to be represented by a wedge in OpenFOAM only one cell thick.

$2 \mathrm{~m}$ is air. The floating cylindrical body under investigation is placed in the exact centre of the tank and has an equilibrium buoyancy position $50 \%$ submerged so that its centre of mass coincides with the still water level at the centre of the tank. For the different free decay experiments, the initial position of the body is displaced vertically from this equilibrium position and is constrained to move only in heave along the tank's central axis. Since the body is constrained to move in heave only, static stability is not an issue.

The next step in the CFD NWT process is the meshing and we can take advantage of the symmetry of the NWT geometry to simplify the process. The tank and the body are axi-symmetric and the body only moves in heave along the tank's central axis; therefore, only one slice of the full circle (Fig. 7) needs to be modelled if symmetric boundary conditions are applied, as implemented in OpenFOAM using the wedge (OpenFOAM, 2013) command. The arc of the wedge spans one degree in angle and is only one cell thick, effectively transforming the three dimensional problem to two dimensions, which significantly reduces the number of cells needed to mesh the domain, considerably speeding up run time.

The tank is meshed with hexahedral cells using OpenFOAM's mesh generator blockMesh. In the horizontal direction there are 1000 cells, with cell grading utilised so that the first cell in the centre of the tank is 100 times smaller than the last cell next to the tank wall. This allows a fine mesh resolution near the body while reducing the total number of cells needed to cover the full width of the tank. In the vertical direction 1000 cells with a uniform height of $5 \mathrm{~mm}$ are used.

Four free decay experiments with varying initial displacements of 5, 10, 20 and $45 \mathrm{~cm}$ were conducted in the NWT. The experiments were simulated for $12 \mathrm{~s}$ and as shown in Fig. 8 (which is a CFD post processing view of the dynamic pressure field in the water after $6 \mathrm{~s}$ of simulation) the generated waves propagating from the oscillating body do not reach the tank wall by the half way mark of the simulation thus will not be reflected back to the body before the end of the simulation and influence the results. The evolution of the floating body's position in time for the four experiments is plotted in Fig. 9.

OpenFOAM calculates the total pressure, as well as the dynamic pressure, in the fluid at each time step and offers functions which integrate these values over the floating body's surface area to give the total force, as well as the dynamic force on the body from the fluid. 


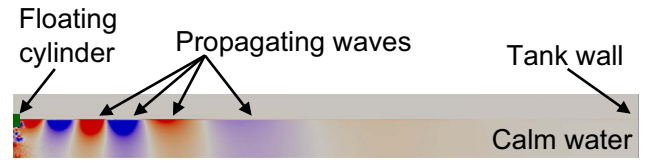

Fig. 8. CFD post process of the dynamic pressure field in the tank after $6 \mathrm{~s}$ of the $12 \mathrm{~s}$ simulation for the $45 \mathrm{~cm}$ initial amplitude free decay experiment.

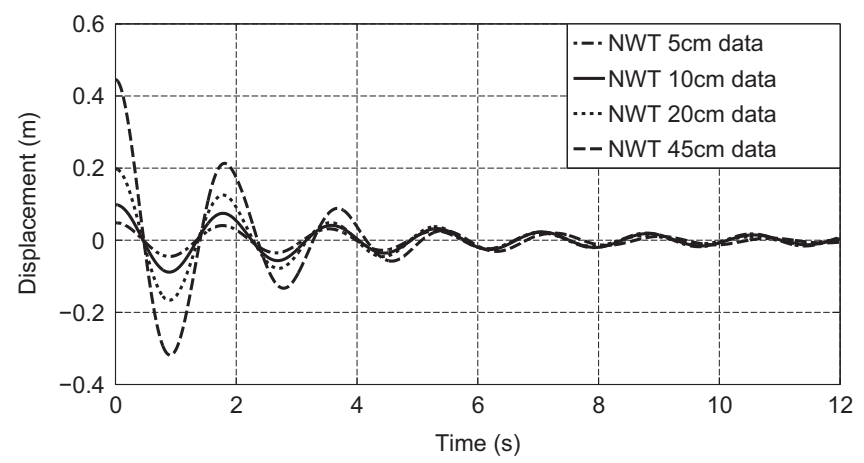

Fig. 9. Simulated results from NWT free decay experiments.

Subtracting dynamic from the total force yields the hydrostatic force on the body from the fluid, which is plotted in Fig. 10, along with the floating body's position for the $45 \mathrm{~cm}$ initial displacement experiment.

To illustrate the inherent nonlinear hydrodynamic effects captured by these CFD simulations, the results in Fig. 9 are normalised against their initial positions and plotted in Fig. 11. Linearity would require these free decay curves to overlay each other when normalised (if linear scaling applies). However, due to nonlinear effects, such as viscosity, vortex shedding (shown in Fig. 12) and time varying wetted body surface area, the normalised NWT responses vary for different initial amplitudes. In total, four experiments, with different initial WEC displacements, provide input data to the parameter identification stage.

\subsection{Parameter identification}

The radiation force convolution integral, Eq. (17), is approximated with a second order state-space subsystem. While this choice provides a good trade-off between accuracy and complexity, the choice also permits the establishment of a distinct dominant pole-pair, which can be tracked through the various models established for different amplitude NWT responses. Also, a distinct pole-pair allows a clear comparison with the results from linear BEM model parameters. Therefore, there are five unknown parameters to be determined for each model $\left(K, a_{0}, a_{1}, b_{1}, m_{\infty}\right)$.

The GA optimisation has been set to have in each generation 300 individuals, 2 elite children that automatically survive to the next generation and about 50\% of children generated with crossover and $50 \%$ with mutation. The algorithm stops when the value of the mean squared error is less than $10^{-15}$ or the number of generations reaches 100 .

Table 1 shows the values determined for these parameters for five different linear models: NWT5, NWT10, NWT20, NWT45 and BEMIM. The first four models' parameters are determined using the methodology outlined in Section 4 and the number in their names represents the initial amplitude of the free decay experiment from which the model is determined; i.e. NWT20 is the model whose parameters are determined from the NWT $20 \mathrm{~cm}$ data in Fig. 9. The fifth model, BEM-IM, uses data generated using a boundary element method (WAMIT) to identify the parameters of the model following the methodology shown in Yu and Falnes (1995).

The results in this table show excellent agreement for the restoring force parameter, $K$, between all five models. The BEM-IM model
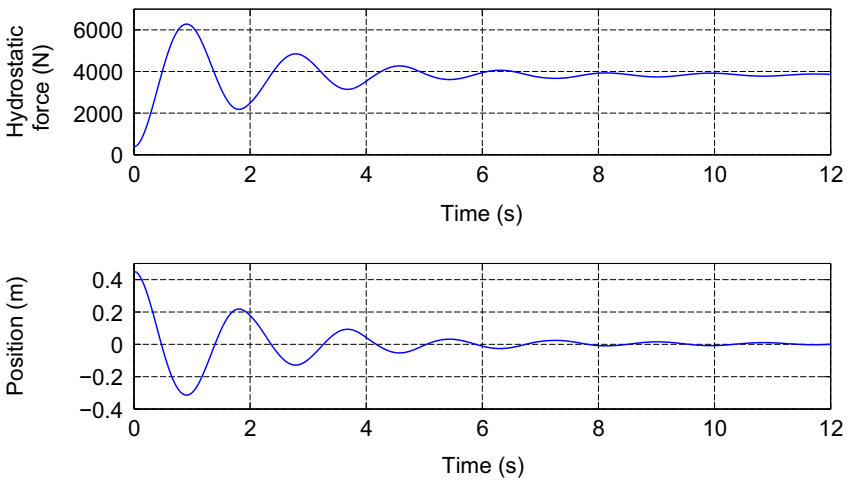

Fig. 10. Time evolution of hydrostatic force and position for the free decay experiment with $45 \mathrm{~cm}$ of initial displacement.

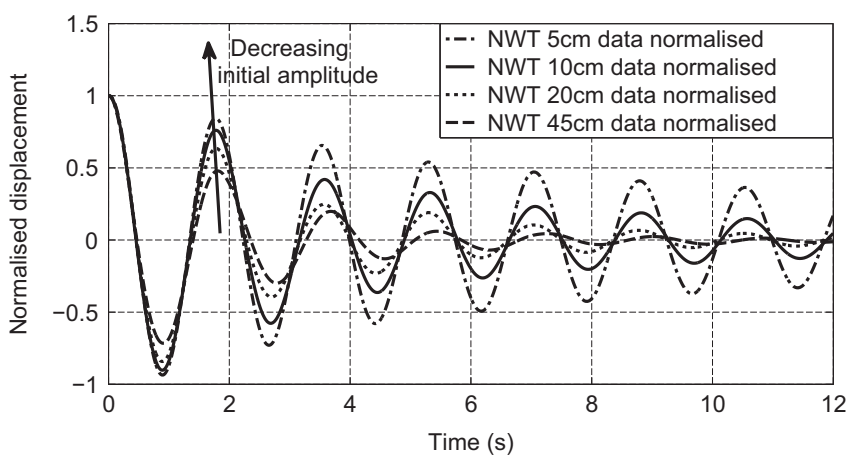

Fig. 11. Simulated results from NWT free decay experiments normalised against their initial position.

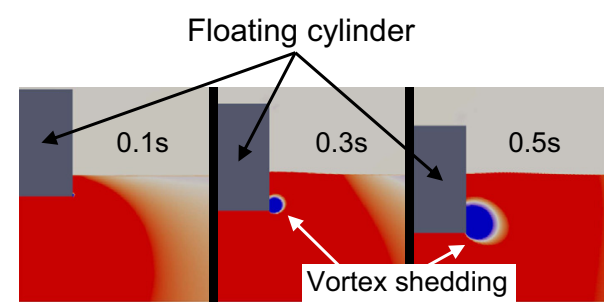

Fig. 12. The dynamic pressure in the fluid around the floating cylinder at $0.1 \mathrm{~s}, 0.3 \mathrm{~s}$ and $0.5 \mathrm{~s}$ showing the creation of a vortex structure from the sharp bottom corner of the cylinder.

Table 1

Estimated model parameters.

\begin{tabular}{llllll}
\hline Model & $\boldsymbol{K}(\mathbf{N} / \mathbf{m})$ & \multicolumn{1}{c}{$\boldsymbol{a}_{\boldsymbol{0}}$} & $\boldsymbol{a}_{\mathbf{1}}$ & \multicolumn{1}{c}{$\boldsymbol{b}_{\mathbf{1}}$} & $\boldsymbol{m}_{\infty}(\mathbf{k g})$ \\
\hline NWT5 & 7704.8 & 6.1647 & 1.9037 & 468.35 & 245.31 \\
NWT10 & 7704.7 & 8.1343 & 2.3840 & 760.35 & 254.77 \\
NWT20 & 7704.8 & 8.5645 & 3.1069 & 1383.9 & 255.99 \\
NWT45 & 7704.4 & 12.6962 & 9.7500 & 5398.7 & 244.44 \\
BEM-IM & 7681.6 & 7.6393 & 1.8582 & 315.82 & 230.20 \\
\hline
\end{tabular}

calculates this parameter as $K=\rho g S$, where $\rho$ is the water density, $g$ is the gravitational constant and $S$ is the body's cross-sectional area in the free surface plane around its equilibrium position. The cylindrical body investigated in this case study has a constant cross-sectional area; therefore, its hydrostatic restoring force should be a linear function of the displacement away from its equilibrium position. This is confirmed by the graph of the restoring force versus floating body position for the $45 \mathrm{~cm}$ initial amplitude NWT experiment plotted in Fig. 13.

The remaining four parameter values vary between the different models, in an attempt to model the significant variation between the 


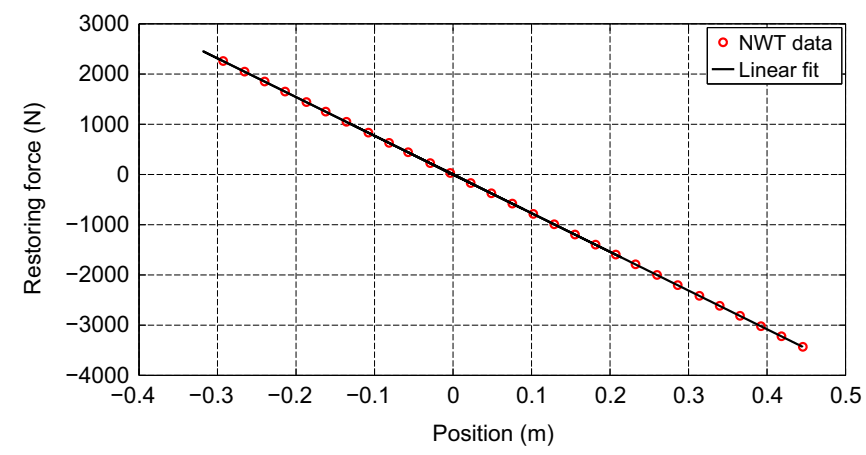

Fig. 13. Restoring force vs. position for the free decay experiment with $45 \mathrm{~cm}$ of initial displacement and its calculated linear fit to obtain the parameter $K$.

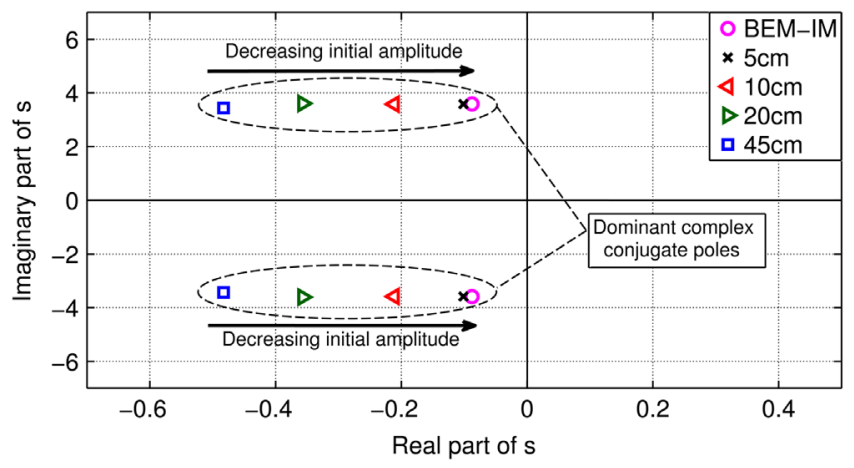

Fig. 14. Location on the complex-plane of the models' dominant poles.

different sets of free response data. These response differences, evident in Fig. 11, show more significant damping for larger initial amplitudes (and subsequent oscillations).

Calculation of the model poles can provide a useful analysis tool. For a stable linear system, the dominant poles are those closest to the imaginary axis and represent slower modes i.e. lower damping effects (the oscillations decay more slowly). Fig. 14 shows that the models whose parameters are determined from the larger initial amplitude conditions do indeed have stronger damping than for models determined from the smaller amplitude conditions, mainly due to nonlinear viscous damping during the initial phase. The arrows in Fig. 14 show this trend.

The only form of energy dissipation from a system described by these linear models is through the radiation term, Eq. (23). All the different dissipative effects modelled by CFD (viscosity and vortex shedding, as well as wave radiation) are all encapsulated into this radiation term of the linear model. Fig. 15 plots the radiation resistances for the different identified models and shows that the models identified from the larger initial amplitude NWT data have larger radiation resistances. Also shown in this figure is that the radiation resistance of the identified models satisfy the properties of Eqs. (10), (11) and (14). Fig. 16 shows that the added mass, Eq. (24), also varies between the different models.

The consequence of the inherent differences between the linear models is that they are representative in the operating (amplitude) region they are identified from and then lose fidelity as they try to predict system behaviour away from this region. This is shown in Fig. 17, which plots the NWT data from the $45 \mathrm{~cm}$ free decay experiment as well as the free decay predictions of the different models when given an initial amplitude of $45 \mathrm{~cm}$. As expected, the NWT45 model predicts closest to the NWT experimental data since it is the exact dataset the model was optimised to fit. The other model's predictions are progressively worse the further their initial amplitude is from the $45 \mathrm{~cm}$ point and the BEM-IM model

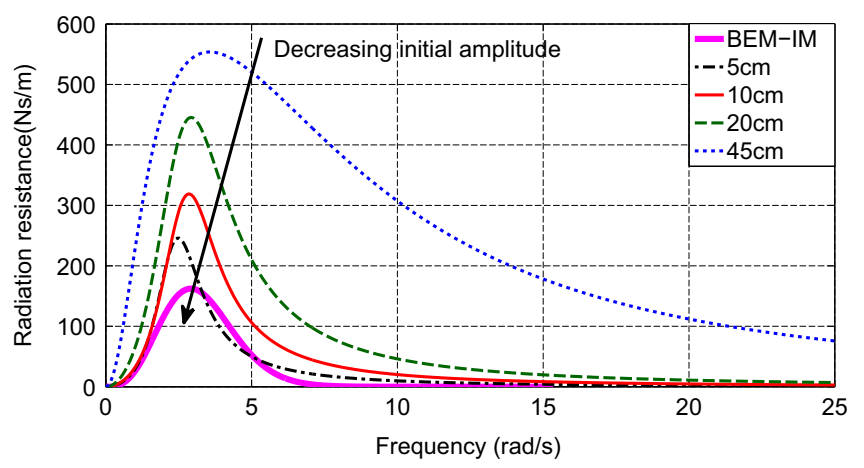

Fig. 15. Radiation resistance of the linear systems identified from the NWT experiments and the radiation resistance calculated by the BEM software WAMIT.

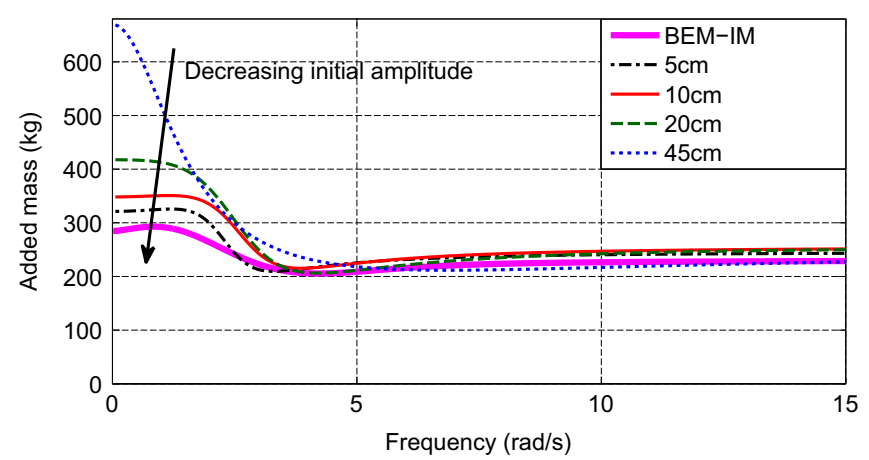

Fig. 16. Added mass of the linear systems identified from the NWT experiments and the added mass calculated by the BEM software WAMIT.

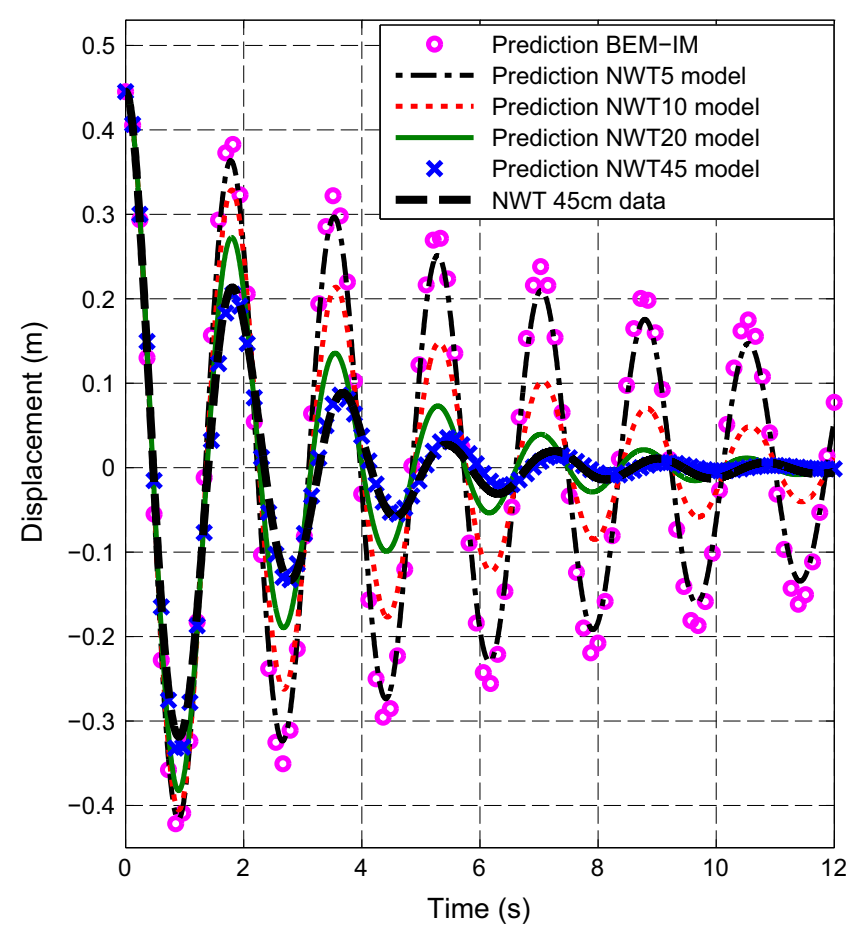

Fig. 17. Plot of predictions made from BEM-IM model, NWT5 model, NWT10 model, NWT25 model, NWT45 model for the same initial displacement $45 \mathrm{~cm}$. Also plotted the NWT simulations with initial displacement of $45 \mathrm{~cm}$.

has the worst fit which is also expected, considering it is effectively linearised about an infinitesmally small deviation around the equilibrium position. 
Table 2

MSE percentage between the predictions by the model NWT5, NWT10, NWT20, NWT45 and the NWT data with initial conditions 5, 10, 20 and $45 \mathrm{~cm}$. The best result for each model is written in bold.

\begin{tabular}{lllll}
\hline Model & \multicolumn{3}{l}{ Initial amplitude } \\
\cline { 2 - 5 } & $\mathbf{5} \mathbf{~ c m}(\%)$ & $\mathbf{1 0} \mathbf{~ c m}(\%)$ & $\mathbf{2 0} \mathbf{~ c m ~ ( \% )}$ & $\mathbf{4 5} \mathbf{~ c m ~ ( \% )}$ \\
\hline NWT5 & $\mathbf{4 . 3 5}$ & 34.0 & 54.0 & 67.6 \\
NWT10 & 43.4 & $\mathbf{7 . 3 2}$ & 28.6 & 46.5 \\
NWT20 & 83.3 & 34.1 & $\mathbf{7 . 5 4}$ & 26.4 \\
NWT45 & 123 & 65.9 & 31.0 & $\mathbf{7 . 9 3}$ \\
BEM-IM & $\mathbf{1 0 . 8}$ & 55.7 & 98.2 & 140.6 \\
\hline
\end{tabular}

To further illustrate the loss in fidelity of the linear models as they try to predict the system behaviour for different amplitudes, Table 2 lists the MSE percentage between the different models predictions and the different NWT experiment data sets. The MSE percentage is defined as

MSE percentage $=100\left(\frac{\sum_{i} \sqrt{\left(y_{N W T}\left(t_{i}\right)-y\left(t_{i}\right)\right)^{2}}}{\sum_{i} \sqrt{y\left(t_{i}\right)^{2}}}\right)$

Table 2 shows that each model predicts well in the response region it was identified from and then predicts progressively worse as the initial amplitude moves further from the initial amplitude of the data that the model used for its identification.

\section{Conclusions}

This paper demonstrates a general framework, shown in Fig. 1 for the identification of linear and nonlinear parametric models from numerical wave tank (NWT) responses, which are determined from the fully nonlinear Navier-Stokes equations. In this paper, we focus exclusively on linear models, for simplicity and clarity, but also to allow a direct comparison with hydrodynamic models based on linear boundary-element methods (BEMs). However, families of representative linear models valid for various oscillation amplitudes could be used to form a composite nonlinear model, following the general philosophy outlined in Smith and Johansen (1997) and Leith and Leithead (1999).

The use of free response tests allows a focus on the state dynamics of the linear system, and the direct comparison of the free response with varying initial condition amplitudes, reveals the breakdown of the linear assumption. This is particularly evident in the normalised responses of Fig. 11, where linear scaling is clearly not valid. As a result, there is considerable variation in the derived linear model parameters and indeed, the representative linear parameters are themselves a compromise over the decay period, since the oscillation amplitude is not constant. However, in normal operational mode, WEC oscillation amplitudes will be reasonably consistent, for a given sea state, allowing the use of representative linear models, tuned for each sea state.

It is also clear from the results (e.g. Fig. 17) that the results obtained from linear boundary-element methods quickly lose fidelity, as the oscillation amplitude becomes significant. However, the convergence of the poles of the models derived through our identification method with those obtained using WAMIT, as the oscillation amplitude asymptotically approaches zero, provides a validation of our modelling approach. While caution must be exercised in extending our conclusions to other WEC geometries, it is reasonable to infer that all osc- illatory WECs are likely to see significant deterioration of the fidelity of BEM-based linear models as the oscillation amplitude grows.

Further research will focus on the development of complete piecewise linear models to cover the whole WEC operational space, the determination of other parametric nonlinear models, and models determined using forced response NWT tests.

\section{Acknowledgement}

This project is funded by Enterprise Ireland and is co-funded by the Irish Government and the European Union under Irelands EU Structural Funds Programme 2007-2013 under Grant EI/CF/2011/ 1320.

\section{References}

Afshar, M.A., 2010. Numerical Wave Generation in Open Foam (Master's thesis). Chalmers University of Technology.

Bhinder, M., Babarit, A., Gentaz, L., Ferrant, P., 2012. Effect of viscous forces on the performance of a surging wave energy converter. In: Proceedings of the Twenty-second (2012) International Offshore and Polar Engineering Conference.

Cathelain, M., 2013. Modelling of a Floating Object in Heave Motion Using a Numerical Wave Tank in Openfoam (Master's thesis). Ecole Centrale de Nantes.

Cummins, W., 1962. The impulse response function and ship motions. Technical Report, DTIC Document.

Furuta, K., Sano, A., Atherton, D., 1988. State Variables Methods in Automatic Control. John Wiley \& Sons, New York.

Gilloteaux, J.-C., 2007. Simulation de mouvements de grande amplitude-application a la recouperation de l'energie des vagues (Ph.D. thesis). Ecole Centrale de Nantes.

Gilloteaux, J.-C., Bacelli, G., Ringwood, J.V., 2008. A non-linear potential model to predict large-amplitudes-motions: application to a multi-body wave energy converter. In: Proceedings of the 10th World Renewable Energy Conference.

Kailath, T., 1980. Linear Systems. Prentice-Hall, Englewood Cliffs, NJ, USA.

Lambert, R.J., 2012. Development of a Numerical Wave Tank Using Openfoam (Master's thesis). Universidade De Coimbra.

Leith, D., Leithead, W., 1999. Analytic framework for blended multiple model systems using linear local models. Int. J. Control 72, 605-619.

Levine, W.S. (Ed.), 2011. The Control Handbook, 2nd ed. CRC Press, Boca Raton, FL, USA.

Li, Y., Yu, Y., 2012. A synthesis of numerical methods for modeling wave energy converter-point absorbers. Renew. Sustain. Energy Rev. 16, 4352-4364.

Ljung, L., 1999. System Identification. Wiley Online Library, Prentice Hall PTR, Upper Saddle River, NJ, USA.

Ljung, L., 2008. Perspectives on system identification. In: Chung, M., Misra, P. (Eds.), Proceedings of the 17th IFAC World Congress: Plenary Papers, Milestone Reports and Selected Survey Papers, Seoul, pp. 47-59 (July).

Maguire, A.E., 2011. Hydrodynamics, Control and Numerical Modelling of Absorbing Wavemakers (Ph.D. thesis). The University of Edinburgh.

Merigaud, A., Gilloteaux, J.-C., Ringwood, J., 2012. A nonlinear extension for linear boundary element methods in wave energy device modelling. In: Proceedings of the ASME 2012 31st International Conference on Ocean, Offshore and Arctic Engineering (OMAE12).

Morison, J.R., O'Brien, P.M., Johnson, J.W., Schaaf, S.A., 1950. The force exerted by surface waves on piles. AIME Petrol. Trans. 189, 149-154.

OpenFOAM, 2013. Openfoam User Guide. Technical Report.

Pérez, T., Fossen, T.I., 2008. Time-vs. frequency-domain identification of parametric radiation force models for marine structures at zero speed. Model. Ident. Control 29, 1-19.

Perez, T., Fossen, T.I., 2011. Practical aspects of frequency-domain identification of dynamic models of marine structures from hydrodynamic data. Ocean Eng. 38, 426-435.

Power, H.M., Simpson, R.J., 1978. Introduction to Dynamics and Control. McGrawHill, New York, USA.

Smith, R.M., Johansen, T.A., 1997. Multiple Model Approaches to Modelling and Control. CRC Press, London, UK.

Taghipour, R., Perez, T., Moan, T., 2008. Hybrid frequency-time domain models for dynamic response analysis of marine structures. Ocean Eng. 35, 685-705.

Tanizawa, K., 2000. The state of the art on numerical wave tank. In: Proceeding of the Fourth Osaka Colloquium on Seakeeping Performace of Ships.

Yu, Z., Falnes, J., 1995. State-space modelling of a vertical cylinder in heave. Appl. Ocean Res. 17, 265-275. 